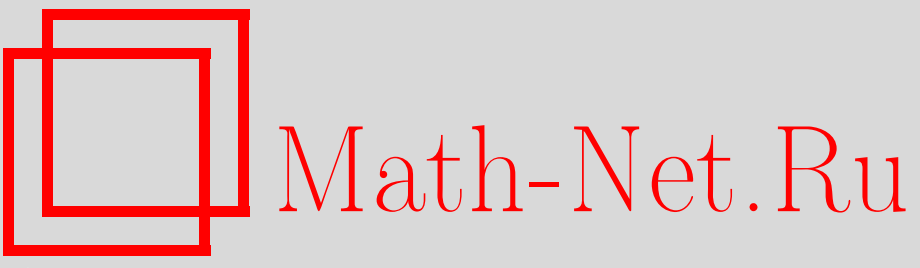

H. Guebbai, S. Lemita, S. Segni, W. Merchela, Difference derivative for an integrodifferential nonlinear Volterra equation, Vestn. Udmurtsk. Univ. Mat. Mekh. Komp. Nauki, 2020, Volume 30, Issue 2, 176-188

DOI: https://doi.org/10.35634/vm200203

Use of the all-Russian mathematical portal Math-Net.Ru implies that you have read and agreed to these terms of use

http://www.mathnet.ru/eng/agreement

Download details:

IP: 3.89 .185 .249

April 26, 2023, 13:50:05 
MSC2010: 45D05, 45J99, 65R20

\author{
(C) H. Guebbai, S. Lemita, S. Segni, W. Merchela
}

\title{
DIFFERENCE DERIVATIVE FOR AN INTEGRO-DIFFERENTIAL NONLINEAR VOLTERRA EQUATION
}

In this article, we propose a new numerical approximation method to deal with the unique solution of the nonlinear integro-differential Volterra equation. We are interested in a very particular form of this equation, in which the derivative of the sought solution appears under the integral sign in a nonlinear manner. Our vision is based on two different approaches: We use the Nyström method to transform the integral into a finite sum using a numerical integration formula, then we use the numerical backward difference derivative method to approach the derivative of our solution. This collocation between two different methods, the first outcome of the numerical processing of integral equations and the second outcome of the numerical processing of differential equations, gives a new nonlinear system for approaching the solution of our equation. We show that the system has a unique solution and that this numerical solution converges perfectly to our solution. A section is dedicated to numerical tests, in which we show the effectiveness of our new vision compared to two methods based only on numerical integration.

Keywords: Volterra integro-differential equation, nonlinear equation, fixed point, numerical derivative, Nyström method.

DOI: $10.35634 / \mathrm{vm} 200203$

\section{Introduction}

The Volterra nonlinear integral equations represent a very great interest in physics and mathematical modeling as history-dependent problems, systems theory, heat conduction and diffusion [1-5]

The analytical and numerical study of its non-differential type has been much studied $[1,6,7]$ compared to the differential type [8,9]. In this paper, we study a particular case of the integro-differential equations type: To find for a given function $f \in C^{1}(a, b)$, a unique solution $u \in C^{1}(a, b)$ such that

$$
\forall t \in[a, b], \quad u(t)=\int_{a}^{t} K\left(t, s, u(s), u^{\prime}(s)\right) d s+f(t) .
$$

The peculiarity and interest of this equation lies in the fact that the derivative of the solution appears under the integral sign in a nonlinear way. Equations of this type are similar to those studied in $[10,11]$, and we can consider this equation as the regular version of the one studied in [12].

The analytical aspect of this equation has been studied in detail by Guebbai et al [10]. Using the hypotheses

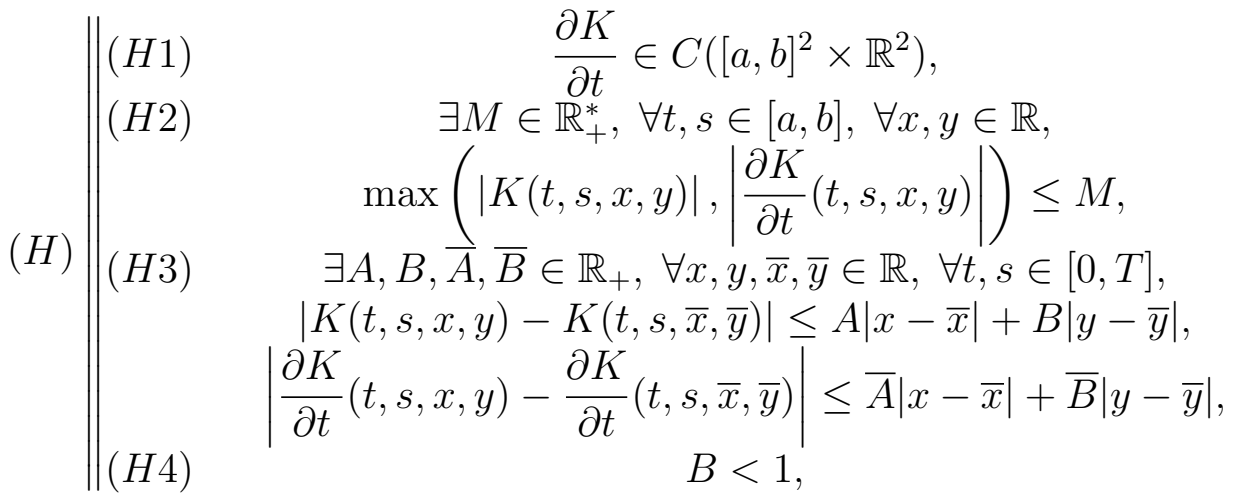


and the fact that

$$
\forall t \in[a, b], \quad u^{\prime}(t)=K\left(t, t, u(t), u^{\prime}(t)\right)+\int_{a}^{t} \frac{\partial K}{\partial t}\left(t, s, u(s), u^{\prime}(s)\right) d s+f^{\prime}(t),
$$

the authors showed that the equation has a unique solution $u \in C^{1}(a, b)$.

In the same paper [10], they use Nyström method [1] to build a numerical approximation method represented in the following nonlinear system:

$$
\begin{aligned}
\forall N \in \mathbb{N}^{*}, h= & \frac{b-a}{N}, t_{j}=a+j h, 0 \leq j \leq N, \\
U_{0} & =f(a), \\
\bar{U}_{0} & =f^{\prime}(a)+K\left(a, a, U_{0}, \bar{U}_{0}\right), \\
U_{i} & =f\left(t_{i}\right)+h \sum_{j=0}^{i} w_{j} K\left(t_{i}, t_{j}, U_{j}, \bar{U}_{j}\right), \quad 1 \leq i \leq N, \\
\bar{U}_{i} & =f^{\prime}\left(t_{i}\right)+K\left(t_{i}, t_{i}, U_{i}, \bar{U}_{i}\right)+h \sum_{j=0}^{i} w_{j} \frac{\partial K}{\partial t}\left(t_{i}, t_{j}, U_{j}, \bar{U}_{j}\right), 1 \leq i \leq N,
\end{aligned}
$$

where, $U_{i}$ approaches $u\left(t_{i}\right), \bar{U}_{i}$ approaches $u^{\prime}\left(t_{i}\right)$ and $\left\{w_{j}\right\}_{j=0}^{N}$ are the weights of an appropriate numerical integration method, supposed to verify:

$$
\exists W>0, \forall N \geq 1, \max _{0 \leq j \leq N}\left|w_{j}\right| \leq W .
$$

But, to obtain the convergence of this numerical scheme, they needed that $A<1$ which is a very restrictive condition.

Recently, Segni et al [11] take up the numerical study of the equation and propose a new numerical scheme that converges under hypotheses $(H)$ only. They start by changing the variable $u$ by the variable $v=u^{\prime}$, which transforms (0.2) into

$$
\begin{aligned}
\forall t \in[a, b], \quad v(t) & =K\left(t, t, f(a)+\int_{a}^{t} v(s) d s, v(t)\right) \\
& +\int_{a}^{t} \frac{\partial K}{\partial t}\left(t, s, f(a)+\int_{a}^{s} v(\tau) d \tau, v(s)\right) d s+f^{\prime}(t) .
\end{aligned}
$$

Once the Nyström method is applied, they get the following numerical scheme:

$$
\begin{aligned}
V_{0} & =f^{\prime}(a)+K\left(a, a, f(a), V_{0}\right) \\
V_{n} & =f^{\prime}\left(t_{n}\right)+K\left(t_{n}, t_{n}, f(a)+h \sum_{i=0}^{n} w_{i} V_{i}, V_{n}\right) \\
& +h \sum_{i=0}^{n} w_{i} \frac{\partial K}{\partial t}\left(t_{n}, t_{i}, f(a)+h \sum_{j=0}^{i} w_{i} V_{i}, V_{i}\right), 1 \leq n \leq N,
\end{aligned}
$$

where, $V_{n}$ approaches $v\left(t_{n}\right)=u^{\prime}\left(t_{n}\right)$ and they use $f(a)+h \sum_{i=0}^{n} w_{i} V_{i}$ to approach

$$
f(a)+\int_{a}^{t_{n}} v(s) d s=u\left(t_{n}\right)
$$


This last method converges perfectly without needing the additional condition $A<1$.

In this work, we propose a new numerical scheme completely different from those proposed in [10] and [11], where we apply the numerical derivative in conjunction with Nyström method. By the way, it is known that the numerical derivative is more usual for partial derivative equations (PDE), but here we give a new vision of numerical derivative to approach the integro-differential equations. On the other hand, as all researchers' work is oriented to construct the weakest conditions that ensure the convergence of their methods, our proposed method is also very efficient, since we need only the hypotheses $(H)$ to confirm its convergence without adding the restrictive condition $A<1$.

\section{$\S 1$. Numerical approximation method}

In this section, we build our numerical technique to approach the solution of equation $(0.1)$ based on two usual numerical principles, the first is the Nyström method based on numerical integration [1,7] and the second is the finite difference method based on numerical derivative [7]. For $N \in \mathbb{N}^{*}$, we define the following subdivision:

$$
h=\frac{b-a}{N}, \quad t_{j}=a+j h, \quad 0 \leq j \leq N .
$$

The formula of the numerical integration is given by:

$$
\forall \xi \in C(a, b), \int_{a}^{b} \xi(t) d t \simeq \tilde{T}(\xi, h)=\frac{h}{2} \xi\left(t_{0}\right)+h \sum_{j=1}^{N} \xi\left(t_{j}\right),
$$

which can be called modified trapezoidal method.

And we use the following numerical derivative, called backward difference, to approach the derivative of our solution

$$
\xi^{\prime}\left(t_{j}\right) \approx \frac{\xi\left(t_{j}\right)-\xi\left(t_{j}-h\right)}{h}=\frac{\xi\left(t_{j}\right)-\xi\left(t_{j-1}\right)}{h}, \quad 1 \leq j \leq N .
$$

Applying these two numerical principles to equations (0.1) and (0.2), we obtain the new system

$$
\begin{aligned}
U_{0}= & f(a), \\
V_{0}= & f^{\prime}(a)+K\left(a, a, U_{0}, V_{0}\right), \\
U_{i}= & f\left(t_{i}\right)+\frac{h}{2} K\left(t_{i}, t_{0}, U_{0}, V_{0}\right)+h \sum_{j=1}^{i} K\left(t_{i}, t_{j}, U_{j}, \frac{U_{j}-U_{j-1}}{h}\right), \quad 1 \leq i \leq N, \\
V_{i}= & f^{\prime}\left(t_{i}\right)+K\left(t_{i}, t_{i}, U_{i}, V_{i}\right)+\frac{h}{2} \frac{\partial K}{\partial t}\left(t_{i}, t_{0}, U_{0}, V_{0}\right) \\
& \quad+h \sum_{j=1}^{i} \frac{\partial K}{\partial t}\left(t_{i}, t_{j}, U_{j}, V_{j}\right), 1 \leq i \leq N,
\end{aligned}
$$

where, $U_{i}$ approaches $u\left(t_{i}\right)$ and $V_{i}$ approaches $u^{\prime}\left(t_{i}\right)$ for $1 \leq i \leq N$.

Our interest is to study the system (1.1)-(1.4): We show that it is well defined and converges to the solution of the equation (0.1). Numerical examples are developed to show its effectiveness compared to systems $(0.3)-(0.6)$ and $(0.7)-(0.8)$.

\subsection{System study}

In the next theorem, we proof the existence and uniqueness of the solution of our new discrete system by using hypotheses $(H)$ only.

Theorem 1. For h sufficiently small and under the hypotheses $(H)$, the system (1.1)-(1.4) has a unique solution. 
P r o o f. First, we have $U_{0}=f(a)$, and by using Banach's fixed point, it is clear that the equation (1.2) has a unique solution $V_{0}$.

We define $\psi_{i}: \mathbb{R} \rightarrow \mathbb{R}, \phi_{i}: \mathbb{R} \rightarrow \mathbb{R}$ for $1 \leq i \leq N$ and $X \in \mathbb{R}$, by:

$$
\begin{aligned}
\psi_{i}(X) & =f\left(t_{i}\right)+h K\left(t_{i}, t_{i}, X, \frac{X-U_{i-1}}{h}\right)+h \sum_{j=1}^{i-1} K\left(t_{i}, t_{j}, U_{j}, \frac{U_{j}-U_{j-1}}{h}\right) \\
& +\frac{h}{2} K\left(t_{i}, t_{0}, U_{0}, V_{0}\right), \\
\phi_{i}(X) & =f^{\prime}\left(t_{i}\right)+K\left(t_{i}, t_{i}, U_{i}, X\right)+h \frac{\partial K}{\partial t}\left(t_{i}, t_{i}, U_{i}, X\right)+h \sum_{j=1}^{i-1} \frac{\partial K}{\partial t}\left(t_{i}, t_{j}, U_{j}, V_{j}\right) \\
& +\frac{h}{2} \frac{\partial K}{\partial t}\left(t_{i}, t_{0}, U_{0}, V_{0}\right) .
\end{aligned}
$$

We have for all $X, Y \in \mathbb{R}$,

$$
\begin{aligned}
\left|\psi_{i}(X)-\psi_{i}(Y)\right| & \leq\left|h K\left(t_{i}, t_{i}, X, \frac{X-U_{i-1}}{h}\right)-h K\left(t_{i}, t_{i}, Y, \frac{Y-U_{i-1}}{h}\right)\right| \\
& \leq\left(h\left(A+\frac{B}{h}\right)\right)|X-Y|=(h A+B)|X-Y|
\end{aligned}
$$

Then, for $h$ sufficiently small, $(h A+B)<1$, so $\psi_{i}$ is a contraction and using Banach's fixed point we obtain that (1.3) has a unique solution $U_{i}$.

In the same way, we get for all $X, Y \in \mathbb{R}$,

$$
\begin{gathered}
\left|\phi_{i}(X)-\phi_{i}(Y)\right| \leq\left|K\left(t_{i}, t_{i}, U_{i}, X\right)-K\left(t_{i}, t_{i}, U_{i}, Y\right)\right|+ \\
+h\left|\frac{\partial K}{\partial t}\left(t_{i}, t_{i}, U_{i}, X\right)-\frac{\partial K}{\partial t}\left(t_{i}, t_{i}, U_{i}, Y\right)\right| \leq(B+h \bar{B})|X-Y| .
\end{gathered}
$$

Also, for $h$ sufficiently small, $(B+h \bar{B})<1$, so $\phi_{i}$ is a contraction and using Banach's fixed point we obtain that (1.4) has a unique solution $V_{i}$.

\subsection{Error analysis}

Now, we show that the solution obtained from our new numerical system converges to the exact solution of the equation (0.1). As we have approached $u^{\prime}\left(t_{i}\right)$ twice, by $V_{i}$ and using its numerical backward difference derivative formula, for this, we define for $i \geq 1$,

$$
\varepsilon_{i}^{1}=u\left(t_{i}\right)-U_{i}, \quad \varepsilon_{i}^{2}=u^{\prime}\left(t_{i}\right)-V_{i}, \quad \varepsilon_{i}^{3}=u^{\prime}\left(t_{i}\right)-\frac{U_{i}-U_{i-1}}{h}, \quad \varepsilon_{i}=\left|\varepsilon_{i}^{1}\right|+\left|\varepsilon_{i}^{2}\right|+\left|\varepsilon_{i}^{3}\right| .
$$

Now, it is said that the method is convergent if

$$
\lim _{h \rightarrow 0} \max _{1 \leq i \leq N}\left(\left|\varepsilon_{i}^{1}\right|+\left|\varepsilon_{i}^{2}\right|\right)=0
$$

this has been demonstrated in $[10,11]$. In this paper, we show that

$$
\lim _{h \rightarrow 0}\left(\max _{1 \leq i \leq N} \varepsilon_{i}\right)=0
$$

Which makes our new method more precise and more efficient. 
For technical reasons, we define local consistency errors, for $i \geq 1$ and $\xi \in C^{1}(a, b)$, by:

$$
\begin{aligned}
\delta_{K}\left(h, t_{i}, \xi\right) & =\int_{a}^{t_{i}} K\left(t_{i}, s, \xi(s), \xi^{\prime}(s)\right) d s \\
& -\frac{h}{2} K\left(t_{i}, t_{0}, \xi\left(t_{0}\right), \xi^{\prime}\left(t_{0}\right)\right)-h \sum_{j=1}^{i} K\left(t_{i}, t_{j}, \xi\left(t_{j}\right), \xi^{\prime}\left(t_{j}\right)\right), \\
\delta_{K_{t}}\left(h, t_{i}, \xi\right) & =\int_{a}^{t_{i}} \frac{\partial K}{\partial t}\left(t_{i}, s, \xi(s), \xi^{\prime}(s)\right) d s \\
& -\frac{h}{2} \frac{\partial K}{\partial t}\left(t_{i}, t_{0}, \xi\left(t_{0}\right), \xi^{\prime}\left(t_{0}\right)\right)-h \sum_{j=1}^{i} \frac{\partial K}{\partial t}\left(t_{i}, t_{j}, \xi\left(t_{j}\right), \xi^{\prime}\left(t_{j}\right)\right),
\end{aligned}
$$

It is clear that our numerical method is consistent with $(0.1)$, i. e.

$$
\forall \xi \in C^{1}(a, b), \lim _{h \rightarrow 0}\left(\max _{1 \leq i \leq N}\left\{\left|\delta_{K}\left(h, t_{i}, \xi\right)\right|,\left|\delta_{K_{t}}\left(h, t_{i}, \xi\right)\right|\right\}\right)=0 .
$$

Indeed, it suffices to show that our modified trapezoidal method is convergent. We recall that the classical trapezoidal formula is given for $N \geq 1$ by

$$
\forall \xi \in C(a, b), \quad T(\xi, h)=\frac{h}{2} \xi\left(t_{0}\right)+h \sum_{j=1}^{N-1} \xi\left(t_{j}\right)+\frac{h}{2} \xi\left(t_{N}\right),
$$

and knowing that

$$
\forall \xi \in C(a, b), \lim _{h \rightarrow 0}\left|\int_{a}^{b} \xi(s) d s-T(\xi, h)\right|=0,
$$

we can conclude that for all $\xi \in C(a, b)$,

$$
\lim _{h \rightarrow 0}\left|\int_{a}^{b} \xi(s) d s-\tilde{T}(\xi, h)\right| \leq \lim _{h \rightarrow 0}\left|\int_{a}^{b} \xi(s) d s-T(\xi, h)\right|+\lim _{h \rightarrow 0} \frac{h}{2}|\xi(b)|=0 .
$$

\section{Theorem 2.}

$$
\lim _{h \rightarrow 0}\left(\max _{1 \leq i \leq N} \varepsilon_{i}\right)=0
$$

P r o o f. For $i \geq 1$, we have:

$$
\begin{aligned}
\varepsilon_{i}^{1} & =\int_{a}^{t_{i}} K\left(t_{i}, s, u(s), u^{\prime}(s)\right) d s-\frac{h}{2} K\left(t_{i}, t_{0}, U_{0}, V_{0}\right)-h \sum_{j=1}^{i} K\left(t_{i}, t_{j}, U_{j}, \frac{U_{j}-U_{j-1}}{h}\right) \\
& =\int_{a}^{t_{i}} K\left(t_{i}, s, u(s), u^{\prime}(s)\right) d s-\frac{h}{2} K\left(t_{i}, t_{0}, U_{0}, V_{0}\right)-h \sum_{j=1}^{i} K\left(t_{i}, t_{j}, u\left(t_{j}\right), u^{\prime}\left(t_{j}\right)\right) \\
& +h \sum_{j=1}^{i} K\left(t_{i}, t_{j}, u\left(t_{j}\right), u^{\prime}\left(t_{j}\right)\right)-h \sum_{j=1}^{i} K\left(t_{i}, t_{j}, U_{j}, \frac{U_{j}-U_{j-1}}{h}\right) \\
& +\frac{h}{2} K\left(t_{i}, t_{0}, u\left(t_{0}\right), u^{\prime}\left(t_{0}\right)\right)-\frac{h}{2} K\left(t_{i}, t_{0}, U_{0}, V_{0}\right) .
\end{aligned}
$$


By using the previous definition of local consistency error, the hypotheses $(H)$ and the equalities $u\left(t_{0}\right)=U_{0}, u^{\prime}\left(t_{0}\right)=V_{0}$ we get for $h$ small enough and $i \geq 1$,

$$
\begin{aligned}
\left|\varepsilon_{i}^{1}\right| & \leq\left|\delta_{K}\left(h, t_{i}, u\right)\right|+h A \sum_{j=1}^{i}\left|\varepsilon_{j}^{1}\right|+h B \sum_{j=1}^{i}\left|\varepsilon_{j}^{3}\right| \\
\left|\varepsilon_{i}^{1}\right| & \leq \frac{\left|\delta_{K}\left(h, t_{i}, u\right)\right|}{1-h A}+\frac{h A}{1-h A} \sum_{j=1}^{i-1}\left|\varepsilon_{j}^{1}\right|+\frac{h B}{1-h A} \sum_{j=1}^{i-1}\left|\varepsilon_{j}^{3}\right|+\frac{h B}{1-h A}\left|\varepsilon_{i}^{3}\right| .
\end{aligned}
$$

In the same way, we get for $h$ small enough and $i \geq 1$,

$$
\begin{aligned}
\left|\varepsilon_{i}^{2}\right| & \leq \frac{\left|\delta_{K_{t}}\left(h, t_{i}, u\right)\right|}{1-(B+h \bar{B})}+\frac{A+h \bar{A}}{1-(B+h \bar{B})}\left|\varepsilon_{i}^{1}\right|+\frac{h \bar{A}}{1-(B+h \bar{B})} \sum_{j=1}^{i-1}\left|\varepsilon_{j}^{1}\right| \\
& +\frac{h \bar{B}}{1-(B+h \bar{B})} \sum_{j=1}^{i-1}\left|\varepsilon_{j}^{2}\right| .
\end{aligned}
$$

Now to estimate the quantity $\left|u^{\prime}\left(t_{i}\right)-\frac{U_{i}-U_{i-1}}{h}\right|$, we begin by:

$$
\begin{gathered}
\frac{U_{i}-U_{i-1}}{h}=\frac{f\left(t_{i}\right)-f\left(t_{i-1}\right)}{h}+\frac{h}{2} \frac{K\left(t_{i}, t_{0}, U_{0}, V_{0}\right)-K\left(t_{i-1}, t_{0}, U_{0}, V_{0}\right)}{h}+ \\
+K\left(t_{i}, t_{i}, U_{i}, \frac{U_{i}-U_{i-1}}{h}\right)+h \sum_{j=1}^{i-1} \frac{K\left(t_{i}, t_{j}, U_{j}, \frac{U_{j}-U_{j-1}}{h}\right)-K\left(t_{i-1}, t_{j}, U_{j}, \frac{U_{j}-U_{j-1}}{h}\right)}{h} .
\end{gathered}
$$

As the function $K(t, s, x, y)$ is differentiable on the parameter $t$, then for $h$ small enough, we can use the following approximation:

$$
\begin{aligned}
\frac{U_{i}-U_{i-1}}{h} & \approx f^{\prime}\left(t_{i}\right)+\frac{h}{2} \frac{\partial K}{\partial t}\left(t_{i}, t_{0}, U_{0}, V_{0}\right)+K\left(t_{i}, t_{i}, U_{i}, \frac{U_{i}-U_{i-1}}{h}\right) \\
& +h \sum_{j=1}^{i-1} \frac{\partial K}{\partial t}\left(t_{i}, t_{j}, U_{j}, \frac{U_{j}-U_{j-1}}{h}\right) .
\end{aligned}
$$

On the other hand, we have:

$$
\begin{aligned}
\varepsilon_{i}^{3} & =\int_{a}^{t_{i}} \frac{\partial K}{\partial t}\left(t_{i}, s, u(s), u^{\prime}(s)\right) d s-\frac{h}{2} \frac{\partial K}{\partial t}\left(t_{i}, t_{0}, u\left(t_{0}\right), u^{\prime}\left(t_{0}\right)\right)-h \sum_{j=1}^{i} \frac{\partial K}{\partial t}\left(t_{i}, t_{j}, u\left(t_{j}\right), u^{\prime}\left(t_{j}\right)\right) \\
& +h \sum_{j=1}^{i-1} \frac{\partial K}{\partial t}\left(t_{i}, t_{j}, u\left(t_{j}\right), u^{\prime}\left(t_{j}\right)\right)-h \sum_{j=1}^{i-1} \frac{\partial K}{\partial t}\left(t_{i}, t_{j}, U_{j}, \frac{U_{j}-U_{j-1}}{h}\right) \\
& +\frac{h}{2} \frac{\partial K}{\partial t}\left(t_{i}, t_{0}, u\left(t_{0}\right), u^{\prime}\left(t_{0}\right)\right)-\frac{h}{2} \frac{\partial K}{\partial t}\left(t_{i}, t_{0}, U_{0}, V_{0}\right) \\
& +h \frac{\partial K}{\partial t}\left(t_{i}, t_{i}, u\left(t_{i}\right), u^{\prime}\left(t_{i}\right)\right)+K\left(t_{i}, t_{i}, u\left(t_{i}\right), u^{\prime}\left(t_{i}\right)\right)-K\left(t_{i}, t_{i}, U_{i}, \frac{U_{i}-U_{i-1}}{h}\right), \\
\left|\varepsilon_{i}^{3}\right| & \leq \frac{\left|\delta_{K_{t}}\left(h, t_{i}, u\right)\right|}{1-B}+\frac{h \bar{A}}{1-B} \sum_{j=1}^{i-1}\left|\varepsilon_{j}^{1}\right|+\frac{h \bar{B}}{1-B} \sum_{j=1}^{i-1}\left|\varepsilon_{j}^{3}\right|+\frac{M}{1-B} h+\frac{A}{1-B}\left|\varepsilon_{i}^{1}\right| .
\end{aligned}
$$


Replacing (1.7) in (1.5), to get for $h$ small enough and $i \geq 1$,

$$
\begin{aligned}
\left|\varepsilon_{i}^{1}\right| & \leq \alpha_{1} \frac{\left|\delta_{K}\left(h, t_{i}, u\right)\right|}{1-h A}+\alpha_{1} \frac{B\left|\delta_{K_{t}}\left(h, t_{i}, u\right)\right|}{(1-B)(1-h A)}+\alpha_{1}\left(\frac{h^{2} B \bar{A}}{(1-B)(1-h A)}+\frac{h A}{1-h A}\right) \sum_{j=1}^{i-1}\left|\varepsilon_{j}^{1}\right| \\
& +\alpha_{1}\left(\frac{h^{2} B \bar{B}}{(1-B)(1-h A)}+\frac{h B}{1-h A}\right) \sum_{j=1}^{i-1}\left|\varepsilon_{j}^{3}\right|+\frac{\alpha_{1} M B}{(1-B)(1-h A)} h^{2}, \\
\alpha_{1} & =\left(1-\frac{h A B}{(1-B)(1-h A)}\right)^{-1},
\end{aligned}
$$

and (1.8) in (1.6), to get for $h$ small enough and $i \geq 1$,

$$
\begin{aligned}
\left|\varepsilon_{i}^{2}\right| & \leq \alpha_{1} \alpha_{2} \frac{\left|\delta_{K}\left(h, t_{i}, u\right)\right|}{1-h A}+\left(\frac{1}{1-(B+h \bar{B})}+\frac{\alpha_{1} \alpha_{2} B}{(1-B)(1-h A)}\right)\left|\delta_{K_{t}}\left(h, t_{i}, u\right)\right| \\
& +\left(\frac{h \bar{A}}{1-(B+h \bar{B})}+\alpha_{1} \alpha_{2}\left(\frac{h^{2} B \bar{A}}{(1-B)(1-h A)}+\frac{h A}{1-h A}\right)\right) \sum_{j=1}^{i-1}\left|\varepsilon_{j}^{1}\right| \\
& +\left(\frac{h \bar{B}}{1-(B+h \bar{B})}\right) \sum_{j=1}^{i-1}\left|\varepsilon_{j}^{2}\right|+\alpha_{1} \alpha_{2}\left(\frac{h^{2} B \bar{B}}{(1-B)(1-h A)}+\frac{h B}{1-h A}\right) \sum_{j=1}^{i-1}\left|\varepsilon_{j}^{3}\right| \\
& +\frac{\alpha_{1} \alpha_{2} M B}{(1-B)(1-h A)} h^{2}, \\
\alpha_{2} & =\frac{A+h \bar{A}}{1-(B+h \bar{B})} .
\end{aligned}
$$

Finally, we replace (1.8) in (1.7), to obtain for $h$ small enough and $i \geq 1$,

$$
\begin{aligned}
\left|\varepsilon_{i}^{3}\right| & \leq \alpha_{1} \alpha_{3} \frac{\left|\delta_{K}\left(h, t_{i}, u\right)\right|}{1-h A}+\left(\frac{1}{1-B}+\frac{\alpha_{1} \alpha_{3} B}{(1-B)(1-h A)}\right)\left|\delta_{K_{t}}\left(h, t_{i}, u\right)\right| \\
& +\left(\frac{h \bar{A}}{1-B}+\alpha_{1} \alpha_{3}\left(\frac{h^{2} B \bar{A}}{(1-B)(1-h A)}+\frac{h A}{1-h A}\right)\right) \sum_{j=1}^{i-1}\left|\varepsilon_{j}^{1}\right| \\
& +\left(\frac{h \bar{B}}{1-B}+\alpha_{1} \alpha_{3}\left(\frac{h^{2} B \bar{B}}{(1-B)(1-h A)}+\frac{h B}{1-h A}\right)\right) \sum_{j=1}^{i-1}\left|\varepsilon_{j}^{3}\right| \\
& +\frac{\alpha_{1} \alpha_{3} M B}{(1-B)(1-h A)} h^{2}+\frac{M}{1-B} h, \\
\alpha_{3} & =\frac{A}{1-B} .
\end{aligned}
$$

Now according to equations (1.8), (1.9) and (1.10) we obtain:

$$
\begin{gathered}
\varepsilon_{i} \leq \frac{\alpha_{1}+\alpha_{1} \alpha_{2}+\alpha_{1} \alpha_{3}}{1-h A}\left|\delta_{K}\left(h, t_{i}, u\right)\right| \\
+\left(\frac{1}{1-B}+\frac{1}{1-(B+h \bar{B})}+\frac{\left(\alpha_{1}+\alpha_{1} \alpha_{2}+\alpha_{1} \alpha_{3}\right) B}{(1-B)(1-h A)}\right)\left|\delta_{K_{t}}\left(h, t_{i}, u\right)\right| \\
+\left(\frac{h \bar{A}}{1-B}+\frac{h \bar{A}}{1-(B+h \bar{B})}+\frac{h^{2} B \bar{A}\left(\alpha_{1}+\alpha_{1} \alpha_{2}+\alpha_{1} \alpha_{3}\right)}{(1-B)(1-h A)}\right.
\end{gathered}
$$




$$
\begin{gathered}
\left.+\frac{h A\left(\alpha_{1}+\alpha_{1} \alpha_{2}+\alpha_{1} \alpha_{3}\right)}{1-h A}\right) \sum_{j=1}^{i-1}\left|\varepsilon_{j}^{1}\right|+\left(\frac{h \bar{B}}{1-(B+h \bar{B})}\right) \sum_{j=1}^{i-1}\left|\varepsilon_{j}^{2}\right| \\
+\left(\frac{h \bar{B}}{1-B}+\frac{h^{2} B \bar{B}\left(\alpha_{1}+\alpha_{1} \alpha_{2}+\alpha_{1} \alpha_{3}\right)}{(1-B)(1-h A)}+\frac{h B\left(\alpha_{1}+\alpha_{1} \alpha_{2}+\alpha_{1} \alpha_{3}\right)}{1-h A}\right) \sum_{j=1}^{i-1}\left|\varepsilon_{j}^{3}\right| \\
+\frac{M B\left(\alpha_{1}+\alpha_{1} \alpha_{2}+\alpha_{1} \alpha_{3}\right)}{(1-B)(1-h A)} h^{2}+\frac{M}{1-B} h .
\end{gathered}
$$

Then,

$$
\begin{aligned}
\varepsilon_{i} & \leq h \gamma \sum_{j=1}^{i-1} \varepsilon_{j}+\left(\frac{1}{1-B}+\frac{1}{1-(B+h \bar{B})}+\frac{\left(\alpha_{1}+\alpha_{1} \alpha_{2}+\alpha_{1} \alpha_{3}\right) B}{(1-B)(1-h A)}\right)\left|\delta_{K_{t}}\left(h, t_{i}, u\right)\right| \\
& +\frac{\alpha_{1}+\alpha_{1} \alpha_{2}+\alpha_{1} \alpha_{3}}{1-h A}\left|\delta_{K}\left(h, t_{i}, u\right)\right|+\frac{M B\left(\alpha_{1}+\alpha_{1} \alpha_{2}+\alpha_{1} \alpha_{3}\right)}{(1-B)(1-h A)} h^{2}+\frac{M}{1-B} h,
\end{aligned}
$$

where

$$
\begin{aligned}
\gamma:=\max & \left(\frac{\bar{A}}{1-B}+\frac{\bar{A}}{1-(B+h \bar{B})}+\frac{h B \bar{A}\left(\alpha_{1}+\alpha_{1} \alpha_{2}+\alpha_{1} \alpha_{3}\right)}{(1-B)(1-h A)}+\frac{A\left(\alpha_{1}+\alpha_{1} \alpha_{2}+\alpha_{1} \alpha_{3}\right)}{1-h A},\right. \\
& \left.\frac{\bar{B}}{1-(B+h \bar{B})}, \frac{\bar{B}}{1-B}+\frac{h B \bar{B}\left(\alpha_{1}+\alpha_{1} \alpha_{2}+\alpha_{1} \alpha_{3}\right)}{(1-B)(1-h A)}+\frac{B\left(\alpha_{1}+\alpha_{1} \alpha_{2}+\alpha_{1} \alpha_{3}\right)}{1-h A}\right) .
\end{aligned}
$$

Applying Theorem 7.1 from [1], we get

$$
\varepsilon_{i} \leq(1+h \gamma)^{i-1} \delta,
$$

where

$$
\begin{array}{r}
\delta:=\max _{1 \leq j \leq i}\left(\left(\frac{1}{1-B}+\frac{1}{1-(B+h \bar{B})}+\frac{\left(\alpha_{1}+\alpha_{1} \alpha_{2}+\alpha_{1} \alpha_{3}\right) B}{(1-B)(1-h A)}\right)\left|\delta_{K_{t}}\left(h, t_{j}, u\right)\right|,\right. \\
\left.\frac{\alpha_{1}+\alpha_{1} \alpha_{2}+\alpha_{1} \alpha_{3}}{1-h A}\left|\delta_{K}\left(h, t_{j}, u\right)\right|, \frac{M B\left(\alpha_{1}+\alpha_{1} \alpha_{2}+\alpha_{1} \alpha_{3}\right)}{(1-B)(1-h A)} h^{2}, \frac{M}{1-B} h\right) .
\end{array}
$$

On the other hand, we have:

$$
(1+h \gamma)^{i-1} \leq\left(1+\frac{(b-a) \gamma}{N}\right)^{N}
$$

and

$$
\lim _{N \rightarrow+\infty}\left(1+\frac{(b-a) \gamma}{N}\right)^{N}<+\infty
$$

Then, $\exists \theta>0$ such that

$$
\forall N \in \mathbb{N}, \max _{1 \leq i \leq N}(1+h \gamma)^{i-1} \leq \theta
$$

which completes the demonstration. 
Table 1: Example 1: Numerical results with $\alpha=e$

\begin{tabular}{llll}
\hline$N$ & $E_{1}$ & $E_{2}$ & $E_{3}$ \\
\hline 200 & $2.64 \mathrm{E}-4$ & $3.01 \mathrm{E}-3$ & $2.97 \mathrm{E}-3$ \\
300 & $1.76 \mathrm{E}-4$ & $2.10 \mathrm{E}-3$ & $1.98 \mathrm{E}-3$ \\
500 & $1.05 \mathrm{E}-4$ & $1.05 \mathrm{E}-3$ & $1.19 \mathrm{E}-3$ \\
1000 & $5.29 \mathrm{E}-5$ & $6.05 \mathrm{E}-4$ & $5.95 \mathrm{E}-4$ \\
1500 & $3.53 \mathrm{E}-5$ & $4.21 \mathrm{E}-4$ & $3.96 \mathrm{E}-4$ \\
\hline
\end{tabular}

\section{\$2. Numerical result}

In this section, to show the effectiveness of our method, we build two numerical examples. As discretization, we use the trapezoidal method, and we mention that the terms $U_{i}$ and $V_{i}$ are not calculated exactly, but they are approached using the Banach's iteration method from our systems (1.3) and (1.4), with the following stopping condition:

$$
\left\|X_{\text {new }}-X_{\text {old }}\right\| \leq 10^{-7}
$$

with a number of iterations that don't exceed 1000. To compare between the methods, we denote the error using our method (1.1)-(1.4) by:

$$
E_{1}=\max _{0 \leq i \leq N}\left\{\left|u\left(t_{i}\right)-U_{i}\right|+\left|u^{\prime}\left(t_{i}\right)-V_{i}\right|\right\}
$$

and by $E_{2}, E_{3}$ the errors obtained when we use the methods described in [10,11] respectively.

Example 1. In the first example, we consider the following equation:

$$
u(t)=\int_{0}^{t} \frac{s e^{-\alpha u^{2}(s)}}{2+t+u^{\prime}(s)^{2}} d s+f(t), \quad \alpha>0, \quad t \in[0,1]
$$

where

$$
f(t)=t-\frac{1}{3+t}\left(\frac{1}{2 \alpha}-\frac{1}{2 \alpha} e^{-\alpha t^{2}}\right)
$$

The exact solution is given by $u(t)=t$. The kernel of this example satisfies $(H)$ with the parameter:

$$
A=\sqrt{\frac{\alpha}{2 e}}
$$

We notice that when $\alpha=e$, which ensures $A<1$, our method is better than those developed in [10] and [11]. But, when we set $\alpha=8 e$ which gives $A>1$, the method (0.3)-(0.6) diverges, unlike our new method which is faster than (0.7)-(0.8).

Example 2. In the second example, we consider the following equation:

$$
u(t)=\frac{1}{2 \pi} \int_{0}^{t} \cos \left(|t-2 s|(t-2 s)+e^{s}-\alpha u(s)+u^{\prime}(s)\right) d s+f(t), \quad \alpha>0, \quad t \in\left[0, \frac{\pi}{2}\right] .
$$


Table 2: Example 1: Numerical results with $\alpha=8 e$

\begin{tabular}{llll}
\hline$N$ & $E_{1}$ & $E_{2}$ & $E_{3}$ \\
\hline 200 & $1.07 \mathrm{E}-4$ & $6.22 \mathrm{E}+1$ & $3.04 \mathrm{E}-3$ \\
300 & $7.15 \mathrm{E}-5$ & $6.34 \mathrm{E}+1$ & $2.03 \mathrm{E}-3$ \\
500 & $4.30 \mathrm{E}-5$ & $6.33 \mathrm{E}+1$ & $1.22 \mathrm{E}-3$ \\
1000 & $2.15 \mathrm{E}-5$ & $6.42 \mathrm{E}+1$ & $6.04 \mathrm{E}-4$ \\
1500 & $1.43 \mathrm{E}-5$ & $6.36 \mathrm{E}+1$ & $4.06 \mathrm{E}-4$ \\
\hline
\end{tabular}

If we take $\alpha=1$, and

$$
\begin{aligned}
f(t) & =\frac{1}{8 \sqrt{\pi}}\left(\sin \left(\frac{t^{2}}{2}\right)\left(\operatorname{FreS}\left(\frac{3}{\sqrt{\pi}} t\right)-\operatorname{FreS}\left(\frac{1}{\sqrt{\pi}} t\right)\right)\right. \\
& \left.-\cos \left(\frac{t^{2}}{2}\right)\left(\operatorname{FreC}\left(\frac{3}{\sqrt{\pi}} t\right)-\operatorname{FreC}\left(\frac{1}{\sqrt{\pi}} t\right)\right)\right) \\
& +\frac{1}{4 \pi t} \sin \left(t^{2}\right)+t e^{t}-4 t^{2}-8 t-8,
\end{aligned}
$$

we get

$$
u(t)=4 t^{2}+8 t+8-t e^{t}
$$

We recall that FreC and FreS denote the Fresnel cosine integral function and the Fresnel sine integral function respectively. The kernel of this example satisfies $(H)$ with the parameter:

$$
A=\frac{\alpha}{2 \pi}=\frac{1}{2 \pi}
$$

But if we take $\alpha=10$, and

$$
\begin{aligned}
f(t) & =\frac{1}{8 \sqrt{\pi}}\left(\sin \left(\frac{t^{2}}{2}\right)\left(\operatorname{FreS}\left(\frac{3}{\sqrt{\pi}} t\right)-\operatorname{FreS}\left(\frac{1}{\sqrt{\pi}} t\right)\right)\right. \\
& \left.-\cos \left(\frac{t^{2}}{2}\right)\left(\operatorname{FreC}\left(\frac{3}{\sqrt{\pi}} t\right)-\operatorname{FreC}\left(\frac{1}{\sqrt{\pi}} t\right)\right)\right) \\
& +\frac{1}{4 \pi t} \sin \left(t^{2}\right)-\frac{1}{9} e^{t}-\left(\frac{2}{5} t^{2}+\frac{2}{25} t+\frac{1}{125}\right),
\end{aligned}
$$

we get

$$
u(t)=\frac{1}{9} e^{t}+\left(\frac{2}{5} t^{2}+\frac{2}{25} t+\frac{1}{125}\right)
$$

In this case,

$$
A=\frac{5}{\pi}
$$

We obtain the same behavior above of the three methods when we vary the parameter $A$. 
Table 3: Example 2: Numerical results with $\alpha=1$

\begin{tabular}{llll}
\hline$N$ & $E_{1}$ & $E_{2}$ & $E_{3}$ \\
\hline 200 & $3.46 \mathrm{E}-4$ & $7.55 \mathrm{E}-4$ & $7.95 \mathrm{E}-4$ \\
300 & $1.53 \mathrm{E}-4$ & $3.21 \mathrm{E}-4$ & $3.54 \mathrm{E}-4$ \\
500 & $1.23 \mathrm{E}-4$ & $1.09 \mathrm{E}-4$ & $1.25 \mathrm{E}-4$ \\
1000 & $4.32 \mathrm{E}-5$ & $9.62 \mathrm{E}-5$ & $8.25 \mathrm{E}-5$ \\
1500 & $2.23 \mathrm{E}-5$ & $6.15 \mathrm{E}-5$ & $5.42 \mathrm{E}-5$ \\
\hline
\end{tabular}

Table 4: Example 2: Numerical results with $\alpha=10$

\begin{tabular}{llll}
\hline$N$ & $E_{1}$ & $E_{2}$ & $E_{3}$ \\
\hline 200 & $5.12 \mathrm{E}-4$ & $7.63 \mathrm{E}+1$ & $8.05 \mathrm{E}-4$ \\
300 & $3.09 \mathrm{E}-4$ & $7.57 \mathrm{E}+1$ & $3.33 \mathrm{E}-4$ \\
500 & $9.36 \mathrm{E}-5$ & $7.48 \mathrm{E}+1$ & $1.12 \mathrm{E}-4$ \\
1000 & $3.52 \mathrm{E}-5$ & $7.34 \mathrm{E}+1$ & $8.52 \mathrm{E}-5$ \\
1500 & $1.98 \mathrm{E}-5$ & $7.28 \mathrm{E}+1$ & $5.51 \mathrm{E}-5$
\end{tabular}

\section{§3. Conclusion}

In this paper we have built a new numerical method to approach the solution of an integrodifferential nonlinear Volterra equation, based on the numerical backward difference derivative.

In practice the numerical derivative is used just for partial deferential equations (PDE), but by applying this numerical technique to our integro-differential equation, we have built a simple and clear approximate system, which is more efficient than those studied in [10] and [11].

As perspectives, according to the simplicity of our method, we will study how to apply this numerical idea for other types of integro-differential equation, as Fredholm equations [13] or equations with weakly singular kernel [12], also the integro-differential equation with higher order [9].

\section{Acknowledgements}

The authors wish to thank Dr. Ammar KHELLAF for his valuable help and remarks.

\section{REFERENCES}

1. Linz P. Analytical and numerical methods for Volterra equations, Society for Industrial and Applied Mathematics, 1985. https://doi.org/10.1137/1.9781611970852

2. Dareiotis K. On finite difference schemes for partial integro-differential equations of Lévy type, Journal of Computational and Applied Mathematics, 2020, vol. 368, 112587. https://doi.org/10.1016/j.cam.2019.112587

3. Behera S., Saha Ray S. An operational matrix based scheme for numerical solutions of nonlinear weakly singular partial integro-differential equations, Applied Mathematics and Computation, 2020, vol. 367, 124771. https://doi.org/10.1016/j.amc.2019.124771

4. Rajagopal N., Balaji S., Seethalakshmi R., Balaji V. S. A new numerical method for fractional order Volterra integro-differential equations, Ain Shams Engineering Journal, 2020, vol. 11, issue 1, pp. 171-177. https://doi.org/10.1016/j.asej.2019.08.004

5. $\mathrm{Xu} \mathrm{D}$. Analytical and numerical solutions of a class of nonlinear integro-differential equations with $L^{1}$ kernels, Nonlinear Analysis: Real World Applications, 2020, vol. 51, 103002. https://doi.org/10.1016/j.nonrwa.2019.103002 
6. Sato T. Sur l'équation intégrale non linéaire de Volterra, Compositio Mathematica, 1953, vol. 11, pp. 271-290. http://www. numdam.org/item/?id=CM_1953_11_271_0

7. Atkinson K., Han W. Theoretical numerical analysis: A functional analysis framework, New York: Springer, 2009. https: //doi .org/10.1007/978-1-4419-0458-4

8. Brunner $\mathrm{H}$. The numerical treatment of Volterra integro-differential equations with unbounded delay, Journal of Computational and Applied Mathematics, 1989, vol. 28, pp. 5-23. https://doi.org/10.1016/0377-0427(89)90318-X

9. Pachpatte B. G. On higher order Volterra-Fredholm integro-differential equation, Fasciculi Mathematici, 2007, no. 37, pp. 35-48.

http://www.math.put.poznan.pl/artykuly/FM37_Pachpatte-wyd02.pdf

10. Guebbai H., Aissaoui M.Z., Debbar I., Khalla B. Analytical and numerical study for an integrodifferential nonlinear Volterra equation, Applied Mathematics and Computation, 2014, vol. 229, pp. 367-373. https://doi.org/10.1016/j.amc.2013.12.046

11. Segni S., Ghiat M., Guebbai H. New approximation method for Volterra nonlinear integro-differential equation, Asian-European Journal of Mathematics, 2019, vol. 12, no. 1, 1950016. https://doi.org/10.1142/S1793557119500165

12. Ghiat M., Guebbai H. Analytical and numerical study for an integro-differential nonlinear Volterra equation with weakly singular kernel, Computational and Applied Mathematics, 2018, vol. 37, issue 4, pp. 4661-4674. https: //doi.org/10.1007/s40314-018-0597-3

13. Pachpatte B.G. On Fredholm type integrodifferential equation, Tamkang Journal of Mathematics, 2008, vol. 39, no. 1, pp. 85-94. https://doi.org/10.5556/j.tkjm.39.2008.48

Received 17.01.2020

Guebbai Hamza, Associate Professor, Department of Mathematics, University 8 Mai 1945, BP 401, Guelma, 24000, Algeria.

E-mail: guebaihamza@yahoo.fr; guebbai.hamza@univ-guelma.dz

Lemita Samir, PhD, Ecole Normale Supérieure de Ouargla, Cité Ennacer, Ouargla, 30000, Algeria.

E-mail: lem.samir@gmail.com

Segni Sami, PhD, Department of Mathematics, University 8 Mai 1945, BP 401, Guelma, 24000, Algeria. E-mail: segnianis@gmail.com; segni.sami@univ-guelma.dz

Merchela Wassim, PhD student, Derzhavin Tambov State University, ul. Internatsional'naya, 33, Tambov, 392000, Russia.

E-mail: merchela.wassim@gmail.com

Citation: H. Guebbai, S. Lemita, S. Segni, W. Merchela. Difference derivative for an integro-differential nonlinear Volterra equation, Vestnik Udmurtskogo Universiteta. Matematika. Mekhanika. Komp'yuternye Nauki, 2020, vol. 30, issue 2, pp. 176-188. 


\section{Х. Геббай, С. Лемита, С. Сегни, В. Мериела}

Разностная производная для интегро-дифференциального нелинейного уравнения Вольтерра

Ключевые слова: интегро-дифференциальное уравнение Вольтерра, нелинейное уравнение, неподвижная точка, численная производная, метод Нистрёма.

УДК 517.988

DOI: $10.35634 / \mathrm{vm} 200203$

В этой статье мы предлагаем новый метод численной аппроксимации для решения единственного решения нелинейного интегро-дифференциального уравнения Вольтерра. Нас интересует особая форма этого уравнения, в которой производная искомого решения появляется под знаком интеграла нелинейным образом. Наше видение основано на двух разных подходах: мы используем метод Нистрёма для преобразования интеграла в конечную сумму, используя формулу численного интегрирования, затем мы используем метод численной обратной разностной производной для приближения к производной нашего решения. Такое сопоставление двух разных методов, первого результата численной обработки интегральных уравнений и второго результата численной обработки дифференциальных уравнений, дает новую нелинейную систему для приближения к решению нашего уравнения. Мы показываем, что система имеет единственное решение и что это численное решение идеально сходится к нашему решению. Раздел посвящен численным тестам, в которых мы показываем эффективность нашего нового видения по сравнению с двумя методами, основанными только на численном интегрировании.

Поступила в редакцию 17.01 .2020

Геббай Хамза, доцент, кафедра математики, Университет 8 мая 1945, 24000, Алжир, г. Гельма, BP 401.

E-mail: guebaihamza@yahoo.fr; guebbai.hamza@univ-guelma.dz

Лемита Самир, PhD, Высшая нормальная школа Уаргла, 30000, Алжир, г. Уаргла, Эннасер.

E-mail: lem.samir@gmail.com

Сегни Сами, PhD, кафедра математики, Университет 8 мая 1945, 24000, Алжир, г. Гельма, ВР 401.

E-mail: segnianis@gmail.com; segni.sami@univ-guelma.dz

Мерчела Вассим, аспирант, кафедра функционального анализа, Тамбовский государственный университет им. Г. Р. Державина, 392000, Россия, г. Тамбов, ул. Интернациональная, 33.

E-mail: merchela.wassim@gmail.com

Цитирование: Х. Геббай, С. Лемита, С. Сегни, В. Мерчела. Разностная производная для интегродифференциального нелинейного уравнения Вольтерра // Вестник Удмуртского университета. Математика. Механика. Компьютерные науки. 2020. Т. 30. Вып. 2. С. 176-188. 